\title{
Gastrointestinal Manifestations in COVID-19 Infection and Its Practical Applications
}

\author{
Amrendra Mandal ${ }^{1}$, Venu Madhav Konala ${ }^{2,3}$, Sreedhar Adapa ${ }^{4}$, Srikanth Naramala ${ }^{5}$, Vijay Gayam ${ }^{1}$ \\ 1. Internal Medicine, Interfaith Medical Center, Brooklyn, USA 2. Hematology and Oncology, Ashland Bellefonte \\ Cancer Center, Ashland, USA 3. Hematology and Oncology, King's Daughters Medical Center, Ashland, USA 4. \\ Nephrology, Kaweah Delta Medical Center, Visalia, USA 5. Rheumatology, Adventist Medical Center, Hanford, USA
}

Corresponding author: Amrendra Mandal, amrendramandal@gmail.com

\begin{abstract}
The latest novel coronavirus (COVID-19) outbreak, which emerged in December 2019 in Wuhan, Hubei, China, is a significant cause of the pandemic. This outbreak is caused by severe acute respiratory syndrome coronavirus 2 (SARS-CoV-2) and is also commonly known as COVID-19. A typical symptom includes cough and fever, but a considerable number of patients can manifest gastrointestinal (GI) symptoms, including diarrhea, which can be the initial presentations and may or may not present with respiratory symptoms or fever. COVID-19 virus may be present in stool samples of patients infected with COVID-19, and angiotensin-converting enzyme 2 (ACE2) is a receptor for this virus, which is substantially present in GI epithelial cells. The wide availability of this receptor facilitates COVID-19 infection to be proactive and multiply in the GI tract. Although no antiviral treatments have been approved, several approaches have been proposed, and at present, optimized supportive care remains the mainstay of therapy. Elective endoscopic procedures should be delayed, but the urgent procedures should be performed as indicated. Due to the rapidly evolving data on COVID-19, it is difficult to keep up with the outpouring of information. We reviewed the mechanisms, clinical manifestation, impact on pre-existing liver diseases, and recommendations endorsed by the several GI societies for the management and prevention of its transmission.
\end{abstract}

Categories: Internal Medicine, Gastroenterology, Infectious Disease

Keywords: covid-19, ace2, gastrointestinal manifestations, pre-existing gi diseases, gi societies

\section{Introduction And Background}

Unexpected pneumonia was observed in early December 2019 in Wuhan, China [1]. Subsequently, a newly emerged coronavirus was labeled as SARS-CoV-2 (severe acute respiratory syndrome coronavirus 2 ) by the Coronavirus Study Group of the International Committee on Taxonomy of Viruses [2-4]. Coronavirus (COVID-19) resulted in a significant pandemic, with the incubation period to be 6.4 days (average), ranging from 2.1-11.1 days [5]. A wide variety of symptomology and radiographic appearances create difficulty for clinicians to identify COVID-19 and differentiate it from other more frequent respiratory infections. The usual symptoms include fever, cough, difficulty in breathing, and myalgia or fatigue, but atypically isolated sudden onset anosmia as well as a loss of taste in COVID-19 infection has also been reported [6-8]. It has also been shown that COVID-19 persists on inanimate surfaces for up to nine days; thus, it can be infective without close contact with an infected person [9]. Interestingly, patients with COVID-19 infection also demonstrated GI manifestations with diarrhea, vomiting, and abdominal pain [10]. Studies have also recognized the presence of COVID-19 virus in anorectal swabs as well as stool samples despite the virus being disappeared in the upper respiratory tract samples [11-13].

The studies demonstrated that the COVID-19 receptor, i.e., angiotensin-converting enzyme 2 (ACE2), is observed to be also expressed in gastrointestinal (GI) epithelial cells [14]. At the same time, these suggest that COVID-19 can be proactive and can significantly multiply in the GI tract. Although no antiviral treatments have been approved so far, several approaches have been proposed. This article aims to review the mechanisms, clinical manifestation, impact on pre-existing digestive diseases, and recommendations endorsed by the several GI societies for the management and prevention of its transmission. We also examine how it could impact gastroenterologists, hepatologists, transplant surgeons, and review the recommendations from different societies to mitigate the infection during the procedures.

\section{Mechanisms of gastrointestinal tract involvement}

Although COVID-19 is transmitted through respiratory droplets and close contact, indirect contact by contaminated inanimate objects also takes part in virus transmission to some extent $[15,16]$. ACE2 has been shown in the previous study as a receptor for different coronaviruses such as SARS-CoV. It has also been shown that COVID-19 uses ACE2 as a viral receptor for the entry process [17]. There are existing data than ACE2 is known to be rich in the epithelial cells of the lungs and GI tracts in humans, which might facilitate the evidence of this possible route for COVID-19 infection [18]. It is highly expressed in the glandular cells of gastric, duodenal, and rectal epithelia, supporting the entry of COVID-19 into the host cells. In a study that 
analyzed COVID-19 patients, 39 (53.4\%) were found to be positive for COVID-19 in stool, with a duration of positive stool ranging between 1 and 12 days [14]. Interestingly, 17 (23.3\%) patients were persistently positive for COVID-19 infection in stool even after a negative polymerase chain reaction (PCR) test in their respiratory specimens. In another study that followed 10 pediatric patients and evaluated their nasopharyngeal and rectal swabs, 8 children were persistently tested positive on rectal swabs even after nasopharyngeal clearance of the virus [19]. Zhang et al. described the presence of viral RNA in the anal swabs and fecal specimens of patients with COVID-19 [11]. Therefore, there is evidence of fecal-oral transmission in COVID-19 infection, and therefore rectal swab may also play a role in establishing a viral clearance.

Furthermore, a cohort study demonstrated a significant enhancement of ACE2 expression in cholangiocytes (59.7\% of cells) as compared with liver cells (2.6\% of cells), indicating that COVID-19 can lead to direct damage of intrahepatic bile ducts. However, the histopathological study of liver tissue from COVID-19 patients did not show viral inclusions in the liver specimens [20]. There may be other possibilities of liver abnormality in COVID-19 patients, such as drug used in the treatment itself or systemic inflammatory response induced by cases of pneumonia [10].

\section{Review \\ Liver abnormalities}

The recent literature suggests that there may be mild-to-moderate injury to the liver, with elevation in aminotransferases, decline in albumin levels, and rise in prothrombin time in a patient infected with COVID-19, whereas up to $60 \%$ of patients previously infected with SARS-CoV had liver impairment [21]. The transaminases, such as aspartate aminotransferase (AST) and alanine transaminase (ALT), are elevated more than total bilirubin in the range of $14-53 \%$ in hospitalized COVID-19 patients [8]. Low serum albumin is the marker of disease severity. In one study, gamma-glutamyl transferase was elevated in 30 (54\%) and elevated alkaline phosphatase levels in 1 (1.8\%) of 56 patients with COVID-19 during hospitalization [22]. The histopathological examination revealed microvesicular steatosis and mild lobular activity in COVID-19 infection [20].

\section{Gastrointestinal manifestation}

Patients who presented with GI symptoms usually have a longer time from the onset of symptoms to hospital admissions than patients without these symptoms (9.0 days vs. 7.3 days). Early symptoms in the majority of COVID-19 patients have fever, myalgia, cough, and sore throat, as manifested with the other acute respiratory virus infections $[23,24]$. The majority of patients with COVID-19 infections present with mild symptoms, and most admitted patients have pneumonia with ground-glass opacities on chest imaging. Diagnosis becomes even more difficult considering the probability of a large number of mild or asymptomatic patients who are not recognized as a COVID-19 infection [19,25]. In one initial retrospective study from Wuhan, GI symptoms, such as diarrhea (2\%-10.1\%), nausea, and vomiting (1\%-3.6\%), were not very common $[23,26]$. However, with evolving studies related to COVID-19, up to 48.5\% (204 patients) reported having GI symptoms at presentation in China. They presented with symptoms such as anorexia, diarrhea, vomiting, and abdominal pain [27].

\section{Patients with underlying digestive diseases}

COVID-19 infection may present with a severe infection in chronic liver diseases such as viral hepatitis B or C [28].

\section{Hepatitis B virus}

In the study of 1,099 patients, 23 (2.1\%) patients had hepatitis B virus (HBV) infection, and severe cases had HBV infection (2.4\% vs. $0.6 \%$ ) than non-severe cases [28]. Currently, there are no available data on the preexisting chronic liver disease, such as non-alcoholic fatty liver disease and alcohol-related liver disease, and their impact on the outcome of COVID-19. It is also not known if COVID-19 infection aggravates cholestasis in those with pre-existing cholestatic liver diseases such as primary biliary cholangitis or primary sclerosing cholangitis or with underlying cirrhosis [22].

\section{Decompensated cirrhosis}

There is little information about the impact of COVID-19 infection in patients with chronic liver disease. Patients with chronic liver disease should be considered for the evaluation of COVID-19 if manifested with hepatic encephalopathy, and patients with hepatic hydrothorax, portopulmonary hypertension, or hepatopulmonary syndrome also need to be considered for aggressive airway management [28].

\section{GI tumors including hepatocellular carcinoma}

There are no data on whether hepatocellular carcinoma (HCC) causes severe COVID-19 infection. However, 
one case series revealed worse COVID-19 outcomes with non-hepatic tumors [29]. Whether patients with other GI cancers are more susceptible to COVID-19 infection than those with non-GI cancers also remains unknown [30]. The American Association for the Study of Liver Diseases (AASLD) recommends continuing the usual HCC surveillance imaging in patients with HCC. A delay of roughly two months may be reasonable for surveillance, as the possible duration of the pandemic cannot be estimated at this time. However, if indicated, HCC treatment is recommended to initiate without further delay due to pandemic [30].

\section{Liver transplantation}

At present, there is no exact information on COVID-19 infection and its effects on decompensated cirrhosis or those in the waiting list for liver transplantation (LT). Almost all Organ Procurement Organizations (OPOs) are currently investigating for COVID-19 and will begin with negative donors; although, the capacity to investigate recipients before the proceeding with transplant may be limited. At present, there are no clear data on the impact of COVID-19 infection on patients with decompensated cirrhosis or those already on the waiting list for the LT. So far, there is no report of COVID-19 infection in transplant recipients [31]. Nevertheless, the recipient might get transmission of COVID-19 infection from a donor during LT, and, thus, donor screening is vital, as demonstrated in the earlier SARS-CoV outbreak [32]. LT surgeons are recommended to follow guidelines provided by the Transplantation Society and also follow the local health department guidelines.

\section{Post-transplant}

The evolving studies related to COVID-19 infection show that the innate immune response may be the primary driver for lung injury due to COVID-19, and, in fact, immunosuppression may be protective [33,34]. However, patients on immunosuppressive agents may have persistent viral detection in post-transplant status with COVID-19 infection and may involve in the community spread as well; thus, these patients need to be monitored to prevent the spread of infection along with monitoring of symptoms [35]. The current guidelines recommend not to decrease immunosuppression and also recommend against stopping mycophenolate for stabilized post-transplant patients with COVID-19 infection [35].

\section{Inflammatory bowel disease}

There are no data to suggest that the patients on biologics and immunosuppressive drugs in patients with inflammatory bowel disease (IBD) are more prone to COVID-19 infection. International organizations such as Crohn's and Colitis Foundation and the International Organization for the Study of Inflammatory Bowel Disease (IOIBD) have issued helpful resources due to rising in COVID-19 infection as it relates to IBD medications [36]. The recommendation is to encourage the patients to stay on their IBD medications such as aminosalicylates. However, reducing the dose of steroids is suggested by IOIBD with a dose of $10 \mathrm{mg}$ or above once daily to avoid adrenal insufficiency. Immunomodulators such thiopurines (azathioprine, 6mercaptopurine, cyclosporine, methotrexate) and tofacitinib (JAK inhibitor) inhibit the immune response to viral infections, and the patients should not stop taking these medications. Biologics and biosimilars, including certolizumab pegol, adalimumab, infliximab, and golimumab, are immune-suppressing drugs, and patients are encouraged to continue taking these medications [36].

\section{Treatment}

Appropriate management strategies for patients with COVID-19 infections are rapidly evolving with therapeutic challenges, and optimal agents to treat an infection or prevent progression to critical illness remain unclear. Pharmacological agents such as convalescent plasma from patients who have recovered from viral infections are being used for patients with COVID-19 infection as there was some reported success during SARS-CoV-1, Middle East respiratory syndrome (MERS), Ebola, and H1N1 influenza [37]. However, the safety and efficacy of recovered convalescent plasma transfusion in symptomatic COVID-19-infected patients have not been established, and, currently, no guideline exists in the USA, but this treatment is tried on a case-to-case basis. Other agents, such as remdesivir, chloroquine, and hydroxychloroquine, are showing some good results in small studies and are currently being used in the USA after the evaluation of cardiac parameters [38-40]. The other adjunctive medications, such as lopinavir/ritonavir, tocilizumab, and corticosteroids, are also being used without many benefits [41-43]. The clinical efficacy and safety for the different agents are still under research, and, at present, optimized supportive care remains the mainstay of therapy.

\section{Practical application}

The primary route of COVID-19 transmission is through aerosolized droplets, with the possibility of fecaloral transmission [44]. Considering that COVID-19 infection can be transmitted from an asymptomatic person and also remains detectable in stool specimens even after negative viral RNA from the lung specimen, the several GI societies have come up with joint guidelines to protect the vulnerable persons in the societies, patients, and healthcare professionals [14]. More than 40\% of all COVID-19 infections may be transmitted before the index case manifesting symptoms [24,45]. Moreover, patients with GI symptoms have a longer time from onset to hospitalizations. Physicians should recognize that GI symptoms, such as diarrhea, maybe a presenting feature of COVID-19, and a high index of suspicion requires earlier at-risk 


\section{Cureus}

patients presenting with GI symptoms.

The AASLD recommended testing for HBV and hepatitis $\mathrm{C}$ virus (HCV) in patients with elevated liver enzymes and monitoring liver function tests if treated with remdesivir and tocilizumab [28]. Caution should be taken regarding the use of investigational medications such as remdesivir, tocilizumab, and hydroxychloroquine for COVID-19 infection in pre-existing liver diseases. HCC surveillance may be delayed roughly to two months in patients with cirrhosis, HBV, and HCV and proceeded with HCC treatment without delay if needed [28].

The joint GI societies strongly recommend considering rescheduling non-urgent endoscopic procedures as these procedures are considered aerosol-generating [46,47]. In general, all elective procedures should be delayed, such as screening and surveillance colonoscopy in asymptomatic patients, endoscopic ultrasound (EUS) for intermediate-risk pancreatic cysts, and motility procedures [47]. However, urgent/emergent procedures should not be delayed, such as GI bleeding treatment, esophageal foreign body impaction, endoscopic retrograde cholangiopancreatography (ERCP) for acute cholangitis, EUS drainage for symptomatic pancreaticobiliary disease, and palliation of GI obstruction [47].

The several COVID-19-related studies reporting about GI manifestation have been compared in Table 1 .

\begin{tabular}{|c|c|c|c|c|c|}
\hline Study & Sample & Nausea & Vomiting & Diarrhea & Anorexia \\
\hline Guan et al. [31] & 1,099 & $55(5.0 \%)$ & $55(5.0 \%)$ & 42 (3.8\%) & \\
\hline Wang et al. [26] & 138 & $14(10.1 \%)$ & $5(3.6 \%)$ & $14(10.1 \%)$ & \\
\hline Xiao et al. [14] & 73 & - & - & 26 (35.6\%) & \\
\hline Pan et al. [27] & 204 & - & $8(3.9 \%)$ & $29(14.2 \%)$ & $83(40.6 \%)$ \\
\hline Lu et al. [48] & 171 & 11 (6.4\%) & - & 15 (8.8\%) & \\
\hline Liu et al. [49] & 137 & - & - & $11(8 \%)$ & \\
\hline Zhou et al. [50] & 141 & $7(3.7 \%)$ & $7(3.7 \%)$ & $9(4.7 \%)$ & \\
\hline
\end{tabular}

TABLE 1: Comparisons of GI manifestation in several studies

GI, gastrointestinal

\section{Conclusions}

In this review, we summarized that GI symptoms may be a presenting feature of COVID-19 infection and that a high index of suspicion requires earlier at-risk patients presenting with GI symptoms. We also summarized the guidelines from the joint GI societies for elective GI procedures as well as urgent or emergent procedures and the impact of COVID-19 infection on the several digestive diseases and their management during a pandemic situation.

\section{Additional Information}

\section{Disclosures}

Conflicts of interest: In compliance with the ICMJE uniform disclosure form, all authors declare the following: Payment/services info: All authors have declared that no financial support was received from any organization for the submitted work. Financial relationships: All authors have declared that they have no financial relationships at present or within the previous three years with any organizations that might have an interest in the submitted work. Other relationships: All authors have declared that there are no other relationships or activities that could appear to have influenced the submitted work.

\section{References}

1. Paules CI, Marston HD, Fauci AS: Coronavirus infections-more than just the common cold . JAMA. 2020, 323:707-708. 10.1001/jama.2020.0757

2. Jiang S, Shi Z, Shu Y, Song J, Gao GF, Tan W, Deyin G: A distinct name is needed for the new coronavirus . Lancet. 2020, 395:949. 10.1016/S0140-6736(20)30419-0

3. Zhu N, Zhang D, Wang W, et al.: A novel coronavirus from patients with pneumonia in China, 2019 . N Engl J Med. 2020, 382:727-733. 10.1056/NEJMoa2001017

4. Gorbalenya AE, Baker SC, Baric RS, et al.: The species severe acute respiratory syndrome-related coronavirus: classifying 2019-nCoV and naming it SARS-CoV-2. Nat Microbiol. 2020, 5:536-544. 
10.1038/s41564-020-0695-z

5. Backer JA, Klinkenberg D, Wallinga J: Incubation period of 2019 novel coronavirus (2019-nCoV) infections among travellers from Wuhan, China, 20-28 January 2020. Euro surveillance. 2020, 25:2000062. 10.2807/1560-7917.ES.2020.25.5.2000062

6. Gane SB, Kelly C, Hopkins C: Isolated sudden onset anosmia in COVID-19 infection. A novel syndrome? . Rhinology. 2020, 58:299-301.

7. Bagheri SHR, Asghari AM, Farhadi M, et al.: Coincidence of COVID-19 epidemic and olfactory dysfunction outbreak. medRxiv. 2020, 2020:20041889. 10.1101/2020.03.23.20041889

8. Chen X, Jiang Q, Ma Z, et al.: Clinical characteristics of hospitalized patients with SARS-CoV-2 and hepatitis B virus co-infection. medRxiv. 2020, 2020:20040733. 10.1101/2020.03.23.20040733

9. Guan GW, Gao L, Wang JW, et al.: Exploring the mechanism of liver enzyme Abnormalities in patients with novel coronavirus-infected pneumonia. Zhonghua Gan Zang Bing Za Zhi. 2020, 28:100-106. 10.3760/cma.j.issn.1007-3418.2020.02.002

10. Gu J, Han B, Wang J: COVID-19: gastrointestinal manifestations and potential fecal-oral transmission. Gastroenterology. 2020, 158:1518-1519. 10.1053/j.gastro.2020.02.054

11. Zhang W, Du RH, Li B, et al.: Molecular and serological investigation of 2019-nCoV infected patients: implication of multiple shedding routes. Emerg Microbes Infect. 2020, 9:386-389. 10.1080/22221751.2020.1729071

12. $\mathrm{Xu} \mathrm{Y,} \mathrm{Li} \mathrm{X,} \mathrm{Zhu} \mathrm{B,} \mathrm{et} \mathrm{al.:} \mathrm{Characteristics} \mathrm{of} \mathrm{pediatric} \mathrm{SARS-CoV-2} \mathrm{infection} \mathrm{and} \mathrm{potential} \mathrm{evidence} \mathrm{for}$ persistent fecal viral shedding. Nat Med. 2020, 26:502-505. 10.1038/s41591-020-0817-4

13. Holshue ML, DeBolt C, Lindquist S, et al.: First case of 2019 novel coronavirus in the United States . N Engl J Med. 2020, 382:929-936. 10.1056/NEJMoa2001191

14. Xiao F, Tang M, Zheng X, Liu Y, Li X, Shan H: Evidence for gastrointestinal infection of SARS-CoV-2. Gastroenterology. 2020, 158:1831-1833. 10.1053/j.gastro.2020.02.055

15. Kampf G, Todt D, Pfaender S, Steinmann E: Persistence of coronaviruses on inanimate surfaces and their inactivation with biocidal agents. J Hosp Infect. 2020, 104:246-251. 10.1016/j.jhin.2020.01.022

16. To KK, Tsang OT, Chik-Yan Yip C, et al.: Consistent detection of 2019 novel coronavirus in saliva . Clin Infect Dis. 2020, 12:ciaa149. 10.1093/cid/ciaa149

17. Hoffmann M, Kleine-Weber H, Schroeder S, et al.: SARS-CoV-2 cell entry depends on ACE2 and TMPRSS2 and is blocked by a clinically proven protease inhibitor. Cell. 2020, 181:271-280. 10.1016/j.cell.2020.02.052

18. Hashimoto T, Perlot T, Rehman A, et al.: ACE2 links amino acid malnutrition to microbial ecology and intestinal inflammation. Nature. 2012, 487:477-481. 10.1038/nature11228

19. Wong SH, Lui RN, Sung JJ: Covid-19 and the digestive system. J Gastroenterol Hepatol. 2020, 35:744-748. 10.1111/jgh.15047

20. Xu Z, Shi L, Wang Y, et al.: Pathological findings of COVID-19 associated with acute respiratory distress syndrome. Lancet Respir Med. 2020, 8:420-422. 10.1016/S2213-2600(20)30076-X

21. Chau TN, Lee KC, Yao H, et al.: SARS-associated viral hepatitis caused by a novel coronavirus: report of three cases. Hepatology. 2020, 39:302-310. 10.1002/hep.20111

22. Zhang C, Shi L, Wang FS: Liver injury in COVID-19: management and challenges . Lancet Gastroenterol Hepatol. 2020, 5:428-430. 10.1016/S2468-1253(20)30057-1

23. Chen N, Zhou M, Dong X, et al.: Epidemiological and clinical characteristics of 99 cases of 2019 novel coronavirus pneumonia in Wuhan, China: a descriptive study. Lancet. 2020, 395:507-513. 10.1016/S01406736(20)30211-7

24. Kim JY, Choe PG, Oh Y, et al.: The first case of 2019 novel coronavirus pneumonia imported Into Korea From Wuhan, China: implication for infection prevention and control measures. J Korean Med Sci. 2020, 35:e61. 10.3346/jkms.2020.35.e61

25. Tang A, Tong ZD, Wang HL, et al.: Detection of novel coronavirus by RT-PCR in stool specimen from asymptomatic child, China. Emerg Infect Dis. 2020, 26:1337-1339. 10.3201/eid2606.200301

26. Wang D, Hu B, Hu C, et al.: Clinical characteristics of 138 hospitalized patients with 2019 novel coronavirus-infected pneumonia in Wuhan, China. JAMA. 2020, 323: 1061-1069. 10.1001/jama.2020.1585

27. Pan L, Mu M, Ren HG, et al.: Clinical characteristics of COVID-19 patients with digestive symptoms in Hubei, China: a descriptive, cross-sectional, multicenter study. Am J Gastroenterol. 2020, 115:766-773. 10.14309/ajg.0000000000000620

28. Clinical best practice advice for hepatology and liver transplant providers during the COVID-19 pandemic: AASLD Expert Panel Consensus Statement. (2020). Accessed: May 14, 2020: https:/www.aasld.org/sites/default/files/2020-03/AASLD-COVID19-ClinicalInsights-3.23.2020-FINALv2.pdf.

29. Liang W, Guan W, Chen R, et al.: Cancer patients in SARS-CoV-2 infection: A nationwide analysis in China . Lancet Oncol. 2020, 21:335-337. 10.1016/S1470-2045(20)30096-6

30. Guidance on Coronavirus Disease 2019 (COVID-19) for Transplant Clinician . (2020). Accessed: March 16, 2020: https://tts.org/23-tid/tid-news/657-tid-update-and-guidance-on-2019-novel-coronavirus-2019-ncovfor-transplant-id-cli....

31. Guan WJ, Ni ZY, Hu Y, et al.: Clinical characteristics of coronavirus disease 2019 in China . N Engl J Med. 2020, 382:1708-1720. 10.1056/NEJMoa2002032

32. Kumar D, Tellier R, Draker R, Levy G, Humar A: Severe acute respiratory syndrome (SARS) in a liver transplant recipient and guidelines for donor SARS screening. Am J Transplant. 2003, 3:977-981. 10.1034/j.1600-6143.2003.00197.x

33. Huang C, Wang Y, Li X, et al.: Clinical features of patients infected with 2019 novel coronavirus in Wuhan, China. Lancet. 2020, 395:497-506. 10.1016/S0140-6736(20)30183-5

34. D'Antiga L: Coronaviruses and immunosuppressed patients: the facts during the third epidemic . Liver Transpl. 2020, 26:832-834. 10.1002/1t.25756

35. Information for transplant professionals and community members regarding 2019 novel coronavirus . (2020). Accessed: May 15, 2020: https://www.myast.org/information-transplant-professionals-and-communitymembers-regarding-2019-novel-coronavirus. 
36. COVID-19 (Coronavirus): what IBD patients should know . (2020). Accessed: May 12, 2020: https://www.crohnscolitisfoundation.org/coronavirus/what-ibd-patients-should-know.

37. Chen L, Xiong J, Bao L, Shi Y: Convalescent plasma as a potential therapy for COVID-19 . Lancet Infect Dis. 2020, 20:398-400. 10.1016/S1473-3099(20)30141-9

38. Wang M, Cao R, Zhang L, et al.: Remdesivir and chloroquine effectively inhibit the recently emerged novel coronavirus (2019-nCoV) in vitro. Cell Res. 2020, 30:269-271. 10.1038/s41422-020-0282-0

39. Gautret P, Lagier JC, Parola P, et al.: Hydroxychloroquine and azithromycin as a treatment of COVID-19: results of an open-label non-randomized clinical trial [Online ahead of print]. Int J Antimicrob Agents. 2020, 10.1016/j.ijantimicag.2020.105949

40. Gao J, Tian Z, Yang X: Breakthrough: chloroquine phosphate has shown apparent efficacy in treatment of COVID-19 associated pneumonia in clinical studies. Biosci Trends. 2020, 14:72-73. 10.5582/bst.2020.01047

41. Cao B, Wang Y, Wen D, et al.: A trial of lopinavir-ritonavir in adults hospitalized with severe Covid-19 . N Engl J Med. 2020, 382:1787-1799. 10.1056/NEJMoa2001282

42. Le RQ, Li L, Yuan W, et al.: FDA Approval Summary: tocilizumab for treatment of chimeric antigen receptor t cell-induced severe or life-threatening cytokine release syndrome. Oncologist. 2018, 23:943-947. 10.1634/theoncologist.2018-0028

43. Shang L, Zhao J, Hu Y, Du R, Cao B: On the use of corticosteroids for 2019-nCoV pneumonia . Lancet. 2020, 395:683-684. 10.1016/S0140-6736(20)30361-5

44. Wu D, Wu T, Liu Q, Yang ZJ: The SARS-CoV-2 outbreak: what we know . Int J Infect Dis. 2020, 94:44-48. 10.1016/j.ijid.2020.03.004

45. He X, Lau EH, Wu P, et al.: Temporal dynamics in viral shedding and transmissibility of COVID-19 . Nat Med. 2020, 26:672-675. 10.1038/s41591-020-0869-5

46. Soetikno R, Teoh AY, Kaltenbach T, Lau JY, Asokkumar R, Cabral-Prodigalidad P, Shergill A: Considerations in performing endoscopy during the COVID-19 pandemic [Online ahead of print]. Gastrointest Endosc. 2020, 10.1016/j.gie.2020.03.3758

47. COVID-19: ASGE updates for members. (2020). Accessed: April 16, 2020 : https://www.asge.org/home/advanced-education-training/covid-19-asge-updates-for-members.

48. Lu X, Zhang L, Du H, et al.: SARS-CoV-2 infection in children. N Engl J Med. 2020, 382:1663-1665. 10.1056/NEJMc2005073

49. Liu K, Fang YY, Deng Y, et al.: Clinical characteristics of novel coronavirus cases in tertiary hospitals in Hubei Province. Chinese Med J. 2020, 133:1025-1031. 10.1097/CM9.0000000000000744

50. Zhou F, Yu T, Du R, et al.: Clinical course and risk factors for mortality of adult inpatients with COVID-19 in Wuhan, China: a retrospective cohort study. Lancet. 2020, 395:1054-1062. 10.1016/S01406736(20)30566-3 